\title{
Suppression of inhomogeneities in images of textured surfaces
}

\author{
Jürgen Beyerer \\ Fernando Puente León \\ Universität Karlsruhe (TH) \\ Institut für Me $\beta$ - und Regelungstechnik \\ Postfach 6980 \\ 76128 Karlsruhe, Germany \\ E-mail: beyerer@mrt.mach.uni-karlsruhe.de
}

\begin{abstract}
Automated visual inspection tasks are frequently concerned with the examination of homogeneously textured surfaces such as fabrics, wallpapers, machined surfaces, and floorcoverings. Often, the images taken from such surfaces are degraded by an intensity inhomogeneity due to the image acquisition process. This inhomogeneity is considered to be an irrelevant and disturbing signal component, which should be suppressed to enhance the desired texture component and to ease a subsequent texture analysis. We show that, especially for textured surfaces, it is not always reasonable to assume a pure multiplicative composition of the texture signal and a disturbing inhomogeneity. We introduce a notion of homogeneity of $n$ 'th degree based on first-order statistics and present image processing methods for the homogenization of first, second, and infinite degree. For the homogenization of second degree, we propose a computationally efficient frequency domain signal processing method with high homogenization performance and low nonlinear distortion. Furthermore, we suggest a high-performance homogenization of the infinite-degree technique that equates the local histograms to a global histogram, which is adapted to the given image data. We compare the proposed homogenization methods visually and quantitatively with the well-known homomorphic filtering technique, which assumes a pure multiplicative inhomogeneity. We demonstrate that our methods achieve much better results for synthetic as well as for realistic images of textured surfaces. (c) 1997 Society of Photo-Optical Instrumentation Engineers.
\end{abstract}

Subject terms: image processing; image enhancement; preprocessing; textured surfaces; homogenization; homomorphic filtering; automated visual inspection.

Paper 17046 received Apr. 12, 1996; accepted for publication Aug. 28, 1996.

\section{Introduction}

Tasks in the context of automated visual inspection of technical surfaces are frequently concerned with textures. Examples are fabrics, wallpapers, machined surfaces, floorcoverings, etc. This paper deals with some preprocessing methods useful to homogenize digital images of objects with homogeneously textured surfaces. For simplicity, we treat only gray-level images, although the ideas described can be easily extended to color or other multichannel images.

We define a "physical surface texture" $\boldsymbol{\tau}(\boldsymbol{\xi})$ consisting of two spatially varying components, which contribute differently to the optically formed intensity texture found on the camera target of an automated inspection system:

$\boldsymbol{\tau}(\boldsymbol{\xi}):=\left[\begin{array}{c}\rho\left(\theta_{i}, \varphi_{i}, \theta_{o}, \varphi_{o}, \boldsymbol{\xi}\right) \\ \zeta(\xi)\end{array}\right]$

where $\boldsymbol{\xi}=(\xi, \eta)^{T}$ are the lateral world coordinates. The first component:

$$
\begin{aligned}
\rho\left(\theta_{i}, \varphi_{i}, \theta_{o}, \varphi_{o}, \boldsymbol{\xi}\right)= & \frac{\mathrm{d} L_{o}\left(\theta_{o}, \varphi_{o}, \boldsymbol{\xi} ; E_{i}\right)}{\mathrm{d} E_{i}\left(\theta_{i}, \varphi_{i}, \boldsymbol{\xi}\right)}, \\
& i: \text { incident, } o: \text { observed, }
\end{aligned}
$$

of $\boldsymbol{\tau}(\boldsymbol{\xi})$ is the bidirectional reflectance-distribution function ${ }^{1}$ (BRDF), which describes the local optical properties of the surface material. It tells how bright a surface element viewed from the $\theta_{o}, \varphi_{o}$ direction will appear, if it is illuminated from the $\theta_{i}, \varphi_{i}$ direction. Mathematically, the BRDF is defined as the ratio of radiance $\mathrm{d} L_{o}$ observed in the $\theta_{o}, \varphi_{o}$ direction and caused by $\mathrm{d} E_{i}$ to the irradiance $\mathrm{d} E_{i}$ caused by an incident flux in the $\theta_{i}, \varphi_{i}$ direction. ${ }^{2}$ The elevation angle $\theta$ and the azimuth $\varphi$ belong to a local spherical coordinate system with a polar axis parallel to the normal vector $\mathbf{n}_{s}(\boldsymbol{\xi})$ of the surface. The second component of $\boldsymbol{\tau}(\boldsymbol{\xi})$ is defined as that part of the surface relief, which can be resolved by the image acquisition system (macrostructure). Note that the 3-D microstructure of the surface, which cannot be spatially resolved, contributes to the BRDF. Here $\zeta(\xi)$ is the height of the macrostructure of the surface at the lateral location $\boldsymbol{\xi}$.

The physical texture $\boldsymbol{\tau}(\boldsymbol{\xi})$ is a comprehensive description of the optically relevant surface properties. Unfortunately, in practice, it is usually impossible to determine both com- 


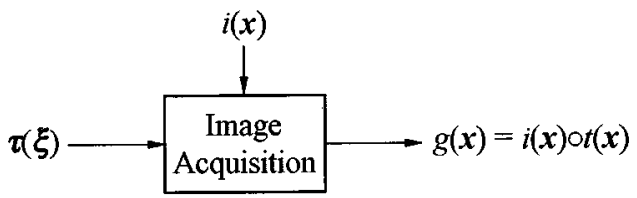

Fig. 1 Signal model.

ponents of $\boldsymbol{\tau}(\boldsymbol{\xi})$ sufficiently fast and at an acceptable expense. Instead, mostly gray-level (or color) images of the surface acquired with CCD cameras are used to accomplish a visual inspection task, although some textural information contained in $\boldsymbol{\tau}(\xi)$ is lost.

For the following considerations, it is convenient to use a simple signal model for gray-level images of textured surfaces (see Fig. 1). Furthermore, the physical texture $\boldsymbol{\tau}(\xi)$ is assumed to be homogeneous. The precise meaning of "homogeneity" is given in Sec. 2.

Within the image acquisition process, the physical texture $\boldsymbol{\tau}(\boldsymbol{\xi})$ is mapped onto the gray-level image $g(\mathbf{x})$, where the vector $\mathbf{x}=(x, y)^{T}$ denotes the camera coordinates. $g(\mathbf{x})$ is thought to be combined of a texture component $t(\mathbf{x})$, which mainly depends on $\boldsymbol{\tau}(\boldsymbol{\xi})$, and of an inhomogeneity $i(\mathbf{x})$, which includes all influences causing $g(\mathbf{x})$ to be inhomogeneous. The inhomogeneity $i(\mathbf{x})$ is considered to be an irrelevant and disturbing signal component, which should be suppressed to enhance the desired texture component $t(\mathbf{x})$ carrying information about $\boldsymbol{\tau}(\boldsymbol{\xi})$.

To find signal processing methods performing a successful separation of both components of $g(\mathbf{x})$, it is necessary to know at least some of the properties distinguishing $i(\mathbf{x})$ and $t(\mathbf{x})$. For example, in Refs. 3 and 4, usually $i(\mathbf{x})$ is assumed to be spatially slowly varying, whereas the texture $t(\mathbf{x})$ has a significant bandpass character. Ideally, the Fourier transforms $I(\mathbf{f})=F\{i(\mathbf{x})\}$ and $T(\mathbf{f})=F\{t(\mathbf{x})\}$ should have disjoint support sets, i.e.,

$\operatorname{supp}\{I(\mathbf{f})\} \cap \operatorname{supp}\{T(\mathbf{f})\}=\varnothing$,

$I(\mathbf{f})=\int_{-\infty}^{\infty} \int_{-\infty}^{\infty} i(\mathbf{x}) \exp \left(-j 2 \pi \mathbf{f}^{T} \mathbf{x}\right) \mathrm{d} \mathbf{x}, \quad \mathbf{f}=\left(f_{x}, f_{y}\right)^{T}$,

$\operatorname{supp}\{I(\mathbf{f})\}=\{\mathbf{f} \| I(\mathbf{f})|\rangle 0\}$.

Moreover, a successful suppression of $i(\mathbf{x})$ requires having a reasonable idea about the functional combination $\circ$ of both components. In Sec. 3, we define $\circ$ such that it is both compatible with our definition of homogeneity and suitable for the construction of powerful homogenization methods.

In many visual inspection tasks, the physical texture is actually homogeneous. If we assume that the signal of interest $t(\mathbf{x})$ results from the physical texture $\boldsymbol{\tau}(\boldsymbol{\xi})$ in a spatially invariant manner, i.e.,

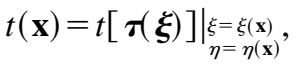

then the homogeneity of $\boldsymbol{\tau}(\boldsymbol{\xi})$ implies that $t(\mathbf{x})$ is also homogeneous.

Many standard signal models for textures such as autoregression (AR), moving average (MA), autoregressive moving average (ARMA), and Markov random field (MRF) models ${ }^{5}$ as well as texture analysis techniques such as cooccurrence methods ${ }^{6}$ or eigenfilters ${ }^{7,8}$ were originally defined for homogeneous or even stationary* textures. Certainly, these methods can be adapted to the cases of inhomogeneous or nonstationary textures, but this also means a drastical increase in computational expense.

If the signal of interest $t(\mathbf{x})$ can be assumed to be homogeneous, it will be advantageous to homogenize the image $g(\mathbf{x})$ to ease a subsequent texture analysis, for example, with the methods mentioned. In addition, homogenization usually causes an image enhancement, which simplifies the visual interpretation of the texture.

\section{Homogeneity}

We now define our notion of homogeneity of $n$ 'th degree. For this purpose, we assume that $g(\mathbf{x})$ is a realization of a stochastic process.

Definition. An image generating stochastic process is called homogeneous of $n$ 'th degree (or $n$-homogeneous for short), if

$\nabla_{x} E\left\{g^{\nu}(\mathbf{x})\right\} \equiv \mathbf{0} \quad \forall 1 \leqslant \nu \leqslant n, \quad \nu \in \mathbf{N}$

holds. Here $E\{\}$ denotes the expectation and $\nabla$ the gradient operator. In other words, a process is called $n$-homogeneous, if its first $n$ moments do not depend on the location $\mathbf{x}$.

Note that this kind of homogeneity affects only firstorder statistics. Second-order and higher order statistics, which describe neighborhood relations of image points, ${ }^{9}$ essentially characterize the appearance of the texture and should therefore not be a subject of a spatial equalization of texture properties. Homogeneity is a necessary condition for stationarity. ${ }^{10}$

Before we deal with the problem of homogenization of an image $g(\mathbf{x})$, we briefly look at some causes of inhomogeneity of $g(\mathbf{x})$ due to the acquisition process, which may occur even if the physical texture $\boldsymbol{\tau}(\boldsymbol{\xi})$ is homogeneous. Examples are

1. spatially varying illumination of the surface

2. $\cos ^{4} \alpha$ dependence of the sensitivity of an imaging system, ${ }^{2}$ ( $\alpha$ is the off-axis angle; see Fig. 2)

3. vignetting

4. spatially varying sensitivity of the camera target

5. spatially varying geometric constellation of the relief $\zeta(\xi)$, the illumination, and the camera lens.

The inhomogeneity arising due to examples, 1 to 4 can be described by a multiplicative signal model: $g(\mathbf{x})=i(\mathbf{x}) t(\mathbf{x})$. Note that $i(\mathbf{x})$ can be suppressed using the well-known homomorphic filtering technique ${ }^{4}$ or by division by a refer-

\footnotetext{
* Our definition of homogeneity is concerned only with first-order statistics. In contrast to this, stationarity means that in addition the secondorder and all higher order statistics are independent of the location $\mathbf{x}$.
} 


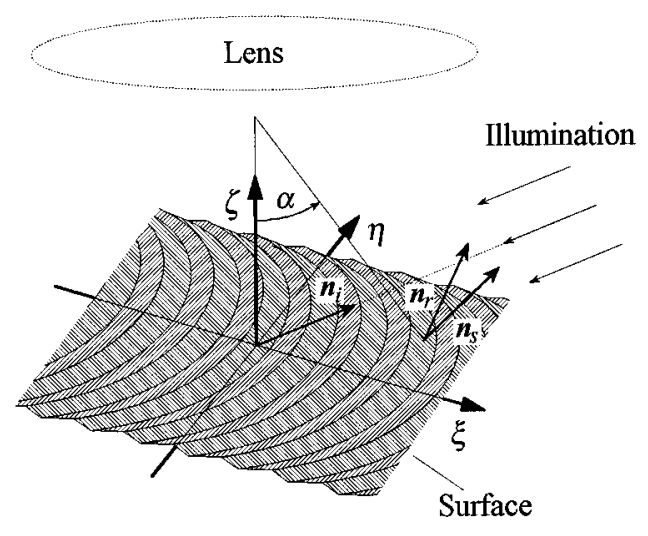

Fig. 2 End-milled surface.

ence image $\propto i(\mathbf{x})$ (Ref. 11). Especially if $\zeta(\boldsymbol{\xi})=$ const, the inhomogeneity due to the acquisition process obeys the multiplicative law.

The situation is different for example 5. With several examples, we make plausible that in the case of example 5, the inhomogeneity should not be explained with a pure multiplicative model, and that a multiplicatively based homogenization like the homomorphic filtering will lead to poor results.

Example. We assume to have an end-milled surface with a physical texture $\boldsymbol{\tau}(\boldsymbol{\xi})=(\text { const, } \zeta(\xi))^{T}$. The BRDF is spatially constant and the relief $\zeta(\xi)$ is 2 -homogeneous. To simplify our discussion, we neglect the inhomogeneity arising due to examples 1 to 4 . The surface is illuminated with spatially constant light parallel to the vector $\mathbf{n}_{i}$ and a camera looks perpendicularly onto the surface (see Fig. 2). We further assume that the BRDF has a narrow mainlobe concentrated around the direction $\mathbf{n}_{r}$ of specular reflection:

$\mathbf{n}_{r}=2 \mathbf{n}_{s} \mathbf{n}_{s}^{T} \mathbf{n}_{i}-\mathbf{n}_{i}$,

where $\mathbf{n}_{s}=\mathbf{n}_{s}(\boldsymbol{\xi})$ is the normal vector of the relief $\zeta(\boldsymbol{\xi})$ at the location $\boldsymbol{\xi}$.

The image $g(\mathbf{x})$ of such an end-milled surface illuminated by parallel light shows a bright stripe in the region where the line

$[\boldsymbol{\xi}, \zeta(\boldsymbol{\xi})]^{T}+\lambda \mathbf{n}_{r}(\boldsymbol{\xi}), \quad \lambda \in \mathbf{R}^{+}$

hits the lens of the camera [see Fig. 6(a) in Sec. 4].

Even though the illumination is spatially constant, the effects of examples 1 to 4 are neglected and the physical texture is 2-homogeneous, the acquired image obviously is inhomogeneous. Our experimental results in Sec. 4 confirm that this kind of inhomogeneity cannot be explained by a pure multiplicative model.

Remarks. First, image processing and texture analysis techniques are not confined to optically acquired images, but can also be applied to any 2-D data regardless of their physical or mathematical background. Examples can be ultrasonic images or tomographically reconstructed images.
If these data consist of a texture of interest mixed with an irrelevant inhomogeneity, a pure multiplicative model will be inappropriate in general.

Second, even if there is a pure multiplication of the texture component and the inhomogeneity, the multiplication may be transformed into different operations by any nonlinearities of the image acquisition process or the image data processing. For example, if there was a logarithmic nonlinearity present, it would transfer a product to a sum.

\section{Homogenization}

In this section, we want to define preprocessing methods suitable to homogenize images $g(\mathbf{x})$ of homogeneous textures $\boldsymbol{\tau}(\boldsymbol{\xi})$. First, we define a neighborhood set $\mathbf{U}$ of the $x, y$ origin, which is small enough that $i(\mathbf{x})$ is approximately constant within every neighborhood set:

$\mathbf{U}(\mathbf{x})=\{\boldsymbol{\chi} \mid \boldsymbol{\chi}=\mathbf{x}+\boldsymbol{\epsilon}, \boldsymbol{\epsilon} \in \mathbf{U}\}$.

On the other side, $\mathbf{U}$ should be significantly larger than the largest details of $t(\mathbf{x})$. This should be possible-at least approximately-because of our assumption that $i(\mathbf{x})$ varies slowly with $\mathbf{x}$ compared with $t(\mathbf{x})$.

Furthermore, we assume the image generating stochastic process to be approximately ergodic in the sense of

$E\left\{g^{\nu}(\mathbf{x})\right\} \approx \frac{1}{|\mathbf{U}|} \iint_{\mathbf{U}} g^{\nu}(\mathbf{x}+\boldsymbol{\epsilon}) \mathrm{d} \boldsymbol{\epsilon} \quad \forall \quad \nu \in \mathbf{N}$,

where $|\mathbf{U}|$ is the area of $\mathbf{U}$. For such a stochastic process, homogeneity of $n$ 'th degree means also that the first $n$ empirical moments on the right-hand side of Eq. (11) are spatially constant.

In practice, often only one image, i.e., only one realization of the image generating process, is available. In this case, Eq. (11) justifies checking for homogeneity and homogenizing the image by using local neighborhood operators. In the following, $\mathscr{H}_{n}\{\}$ denotes a local neighborhood operator making an image $n$-homogeneous.

\subsection{Homogenization of First Degree}

Since 1-homogeneity requires that the expectation of $g(\mathbf{x})$ does not depend on $\mathbf{x}$, a corresponding homogenization affecting exclusively the first moment is reasonable, if $i(\mathbf{x})$ and $t(\mathbf{x})$ are combined additively:

$g(\mathbf{x})=i(\mathbf{x})+t(\mathbf{x})$.

The homogenization can be accomplished by linear highpass filtering:

$t(\mathbf{x}) \approx \mathscr{H}_{1}\{g(\mathbf{x})\}=g(\mathbf{x})-\hat{i}(\mathbf{x}), \quad \hat{i}(\mathbf{x})=\operatorname{LP}\{g(\mathbf{x})\}$.

The linear low-pass filtering $\operatorname{LP}\{\}$ can be carried out by weighted averaging over the neighborhood $\mathbf{U}(\mathbf{x})$, for example. Here $i(\mathbf{x})$ represents the local mean gray value of $g(\mathbf{x})$. 


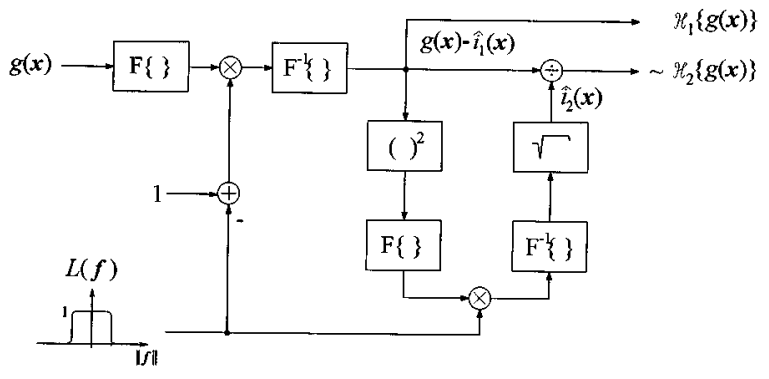

Fig. 3 Computation of $\mathscr{H}_{1}\{\}$ and $\mathscr{H}_{2}\{\}$ in the frequency domain.

\subsection{Homogenization of Second Degree}

Since 2-homogeneity requires the first two moments of $g(\mathbf{x})$ to be independent of $\mathbf{x}$, a corresponding homogenization is reasonable for

$g(\mathbf{x})=i_{1}(\mathbf{x})+i_{2}(\mathbf{x}) t(\mathbf{x})$,

where $i(\mathbf{x})$ has been split in two spatially slowly varying components $i_{1}(\mathbf{x})$ and $i_{2}(\mathbf{x})$, where $i_{1}(\mathbf{x})$ denotes the local mean gray value and $i_{2}(\mathbf{x})$ the local contrast. A homogenization of second degree can be accomplished by

$t(\mathbf{x}) \approx \mathscr{H}_{2}\{g(\mathbf{x})\}=\frac{g(\mathbf{x})-\hat{i}_{1}(\mathbf{x})}{\hat{i}_{2}(\mathbf{x})}$ const.

The subtraction of $\hat{i}_{1}(\mathbf{x})$ leads to an homogenization of first degree and the division by $\hat{i}_{2}(\mathbf{x})$ homogenizes the second moment of $g(\mathbf{x})$. Equation (15) is a special case of the so-called Wallis-operator ${ }^{12,13}$ :

$\frac{b s}{b \hat{i}_{2}(\mathbf{x})+s}\left[g(\mathbf{x})-\hat{i}_{1}(\mathbf{x})\right]+\beta m+(1-\beta) \hat{i}_{1}(\mathbf{x})$,

where $m$ and $s$ are constants, and $b \in[3,25]$ and $\beta \in[0,0.4]$ are tuning parameters. It is used as an enhancement tool for scenic images. Except for an additive constant, Eq. (16) turns into Eq. (15) for $\beta=1$ and $b \rightarrow \infty$. These untypical parameter values lead to an undesired enhancement of subtle details at the expense of principle image features in scenic data, ${ }^{14}$ but are a reasonable choice for images of textured surfaces.

We propose to compute $\mathscr{H}_{2}\{\}$ efficiently in the frequency domain. For that purpose, we use a linear lowpass $\operatorname{LP}\{\}$ to obtain $\hat{i}_{1}(\mathbf{x})$ and $\hat{i}_{2}(\mathbf{x})$ :

$\hat{i}_{1}(\mathbf{x})=\operatorname{LP}\{g(\mathbf{x})\}$,

$\hat{i}_{2}(\mathbf{x})=\left(\operatorname{LP}\left\{\left[g(\mathbf{x})-\hat{i}_{1}(\mathbf{x})\right]^{2}\right\}\right)^{1 / 2}$.

Except for a constant factor, Eq. (18) can be interpreted as an estimate of the local standard deviation of $g(\mathbf{x})$. Then, $\mathscr{H}_{2}\{\}$ can be implemented according to Fig. 3 .

For digital images, the Fourier transforms are done by using a fast Fourier transform (FFT) algorithm. ${ }^{15}$ In addition, $\mathscr{H}_{1}\{g(\mathbf{x})\}$ is also generated in Fig. 3. The low-pass

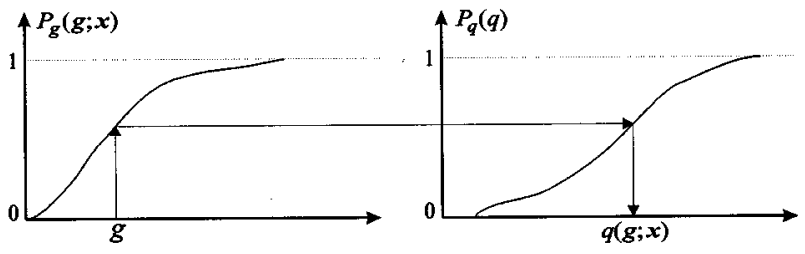

Fig. 4 Space-variant mapping of the gray values $g$ onto $q(g ; \mathbf{x})$.

system function $L(\mathbf{f})$ must be chosen taking the different frequency domain supports of $i(\mathbf{x})$ and $t(\mathbf{x})$ into account.

Note that homomorphic filtering of $\mathscr{H}_{1}\{g(\mathbf{x})\}$ is not appropriate to generate $\mathscr{H}_{2}\{g(\mathbf{x})\}$, because $g(\mathbf{x})-\hat{i}_{1}(\mathbf{x})$ is spatially zero mean. To avoid negative arguments of the logarithm, $g(\mathbf{x})-\hat{i}_{1}(\mathbf{x})$ could be raised by a positive constant. Unfortunately, the resulting image would no longer be a purely multiplicative composition of $i_{2}(\mathbf{x})$ and $t(\mathbf{x})$.

Experimentally, we found that the visually most relevant inhomogeneity is concerned with spatial fluctuations of the mean gray value and the contrast. Thus, a 2-homogenization often is sufficient for a homogeneous visual appearance of the texture. For the other cases, where a still higher homogeneity level is desired, we recommend performing a homogenization of infinite degree.

\subsection{Homogenization of Infinite Degree}

Homogenization of infinite degree requires that all moments are independent of $\mathbf{x}$. This is equivalent to the requirement that all local probability density functions (pdf's) $p_{g}(g ; \mathbf{x})$ are equal to a global pdf $p_{q}(q)$ not depending on $\mathbf{x}$. Therefore, homogenization of infinite degree can be achieved by equating all local pdf's.

We wish to map the gray values $g$ onto values $q(g ; \mathbf{x})$ such that $q(g ; \mathbf{x})$ is distributed according to $p_{q}(q)$. Since the cumulative distribution functions (cdf's) $P_{q}(q)$ and $P_{g}(g ; \mathbf{x})$ must be equal at $q(g ; \mathbf{x})$ (Ref. 4), we obtain the desired mapping as

$$
\begin{aligned}
P_{q}(q(g ; \mathbf{x})) & =\int_{-\infty}^{q(g ; \mathbf{x})} p_{q}(\beta) \mathrm{d} \beta \\
& ! \int_{-\infty}^{g} p_{g}(\beta ; \mathbf{x}) \mathrm{d} \beta=P_{g}(g ; \mathbf{x}) \\
& \Rightarrow q(g ; \mathbf{x}) \\
& =P_{q}^{-1}\left(P_{g}(g ; \mathbf{x})\right),
\end{aligned}
$$

where $P_{g}(g ; \mathbf{x})$ performs a space-variant transformation of $g$ onto a random variable uniformly distributed on $[0,1]$, which $P_{q}^{-1}()$ then transforms onto a random variable distributed according to $p_{q}(q)$ (see Fig. 4).

To apply Eq. (19) to digital images, we must consider that the gray values as well as the locations $\mathbf{x}$ are discrete. Since we assume a local ergodicity according to Eq. (11), the local pdf's can be estimated with local histograms $h(g ; \mathbf{x}, \mathbf{U})$ and local cumulative histograms $H(g ; \mathbf{x}, \mathbf{U})$ :

$h(g ; \mathbf{x}, \mathbf{U})=\frac{1}{|\mathbf{U}|} \sum_{\epsilon \in \mathbf{U}} \delta_{g(\mathbf{x}+\boldsymbol{\epsilon})}^{g}$, 
$H(g ; \mathbf{x}, \mathbf{U})=\sum_{\gamma=g_{1}}^{g} h(\gamma ; \mathbf{x}, \mathbf{U})$,

$g \in\left\{g_{1}, g_{2}, \ldots, g_{G}\right\}, \quad \delta_{a}^{b}= \begin{cases}1 & \text { for } a=b \\ 0 & \text { for } a \neq b\end{cases}$

Now, the neighborhood set $\mathbf{U}$ is discrete and consists of $|\mathbf{U}|$ elements. An approximate realization of the transform of Eq. (19) is

$$
\mathscr{H}_{\infty}\{g(\mathbf{x}) ; H(g)\}=\underset{\gamma \in\left\{g_{1}, \ldots, g_{G}\right\}}{\arg \min }\{|H(\gamma)-H(g ; \mathbf{x}, \mathbf{U})|\},
$$

where $H(g)$ denotes an arbitrary global cumulative histogram to which all local gray-value distributions are to be equal. ${ }^{16}$

So far, nothing is really new regarding $\mathscr{H}_{\infty}\{\}$. Local histogram manipulations are treated for example in Refs. 17 and 18. However, the global histogram $h(g)$ is chosen rather arbitrarily without any reference to the processed data.

With the aim to keep nonlinear distortions low and to achieve a high-homogenization performance, we propose to apply $\mathscr{H}_{\infty}\{\}$ on $\mathscr{H}_{2}\{g(\mathbf{x})\}$ and to define $H(g)$ as

$H(g):=H_{2}(g)=\sum_{\gamma=g_{1}}^{g} h_{2}(\gamma)$,

$h(g):=h_{2}(g)=\frac{1}{N^{2}} \sum_{\mathbf{x} \in \operatorname{supp}\{g(\mathbf{x})\}} \delta_{\mathscr{H}_{2}\{g(\mathbf{x})\}}^{g}$,

for an image of $N \times N$ pixels. Thus the local histograms of $\mathscr{H}_{2}\{g(\mathbf{x})\}$ are equated to the global histogram of $\mathscr{H}_{2}\{g(\mathbf{x})\}$. The successive application of $\mathscr{H}_{2}\{\}$ and $\mathscr{H}_{\infty}\{\}$ can be interpreted as a division of labor, resulting in reduced nonlinear distortions compared with the direct application of $\mathscr{H}_{\infty}\{\}$ on $g(\mathbf{x})$. This can be explained by a smaller modulation of the nonlinear operator $\mathscr{H}_{\infty}\{\}$, when the image that has to be homogenized is already 2-homogeneous, and by the fact that the global histogram is based on the given data.

In other cases, it could be desirable to obtain an image that is not only homogeneous, but whose higher order moments have certain given values. For example, one may want to obtain a homogenized image that, in addition, has no skewness. For this purpose, we suggest performing a homogenization of infinite degree using an Edgeworth approximation of the desired global histogram (see Sec. 6). This approximation depends explicitly on the expectation, the standard deviation, the skewness, and the kurtosis of the gray values. By using the Edgeworth series, adaptation to the given data (to keep nonlinear distortions low) as well as a specific manipulation of the mentioned features can be partly combined.

In the following section, the proposed methods and the homomorphic filtering are compared with regard to homogenization performance and nonlinear distortions.

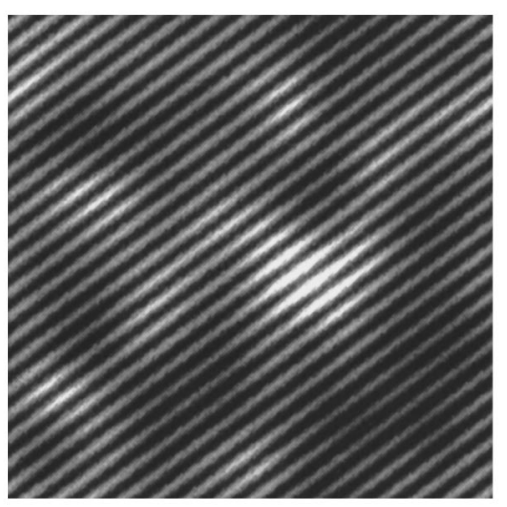

Fig. 5 Harmonic distortion test image.

\section{Experimental Results}

In this section, experimental results of the discussed homogenization methods are presented and contrasted with the homomorphic filtering, which is often the preferred method in the image processing literature for performing a compensation of illumination inhomogeneities. ${ }^{4}$

To compare the performance of the presented homogenization methods, an indicator was developed that enables measuring the inhomogeneity level of an image. The inhomogeneity indicator is based on the computation of distance measures between local cumulative histograms at different scales. Its definition is given in Sec. 7 .

However, the homogeneity measure cannot be the only criterion when evaluating the performance of the homogenization methods. Suppose that a homogenization operation leads to a trivial result, e.g., a binary image. The image may be ideally homogeneous, but its contents would have been obviously heavily distorted. To quantify such undesired effects, harmonic distortions were evaluated as well. For this purpose, a test image was created, which is shown in Fig. 5. In Sec. 8, the definition of the measure of the harmonic distortion and indications of the test image generation are given.

The gray-level images throughout this paper are digitized with $N \times N=256 \times 256$ pixels, $\Delta x=\Delta y$, and 8-bit gray levels. For all operators implemented in the space domain, a mask size of $21 \times 21$ was chosen. The low-pass filter implemented in the frequency domain was chosen to be a Gaussian:

$L(\mathbf{f})=\exp \left[-\frac{1}{2} \frac{\|\mathbf{f}\|^{2}}{\sigma^{2}}(N \Delta x)^{2}\right]$.

To achieve similar transfer characteristics for both implementations, we set $\sigma=12$. For the homomorphic filtering we used $1-L(\mathbf{f})$ for all frequencies $\mathbf{f} \neq \mathbf{0}$, and 1 for $\mathbf{f}=\mathbf{0}$ as the linear system function multiplied with $F\{\log [g(\mathbf{x})]\}$. Because the algorithm is formulated in the Fourier domain, it is easy to adapt specifically the system function $L(\mathbf{f})$ to given spectral characteristics of $i(\mathbf{x})$ and $t(\mathbf{x})$. But in most practical cases, the choice of a rotationally symmetrical $L(\mathbf{f})$, as in Eq. (25), will be sufficient. To avoid artifacts at the image border, a 16-pixel-wide border area was eliminated from the resulting images. 
Table 1 Computation results of the harmonic distortion.

\begin{tabular}{lc}
\hline \hline & $\begin{array}{c}\text { Harmonic } \\
\text { Distortion (\%) }\end{array}$ \\
\hline Homomorphic filtering & 0.0 \\
$\mathscr{H}_{1}\{\}$ computed in the frequency domain & 0.0 \\
$\mathscr{H}_{2}\{\}$ computed in the space domain & 0.4 \\
$\mathscr{H}_{2}\{\}$ computed in the frequency domain & 0.0 \\
$\mathscr{H}_{\infty}\left\{; H_{\text {uni }}(g)\right\}$ & 10.7 \\
$\mathscr{H}_{\infty}\left\{\mathscr{H}_{2}\{\} ; H_{2}(g)\right\}$ & 2.6 \\
\hline \hline
\end{tabular}

In this section, two variants of the homogenization of infinite degree are discussed: $\mathscr{H}_{\infty}\left\{; H_{\text {uni }}(g)\right\}$ denotes that the cumulative histogram of a uniform distribution is used, and $\mathscr{H}_{\infty}\left\{\mathscr{H}_{2}\{\} ; H_{2}(g)\right\}$ denotes the choice proposed in Sec. 3.3 according to Eqs. (23) and (24).

Table 1 shows the computation results for the harmonic distortion. We can see that both operators implemented in the frequency domain lead to practically distortion-free results. Particularly, a significant improvement regarding the harmonic distortion could be achieved by the frequency domain implementation of the $\mathscr{H}_{2}\{\}$ operator. The homomorphic filtering also leads to good results, although it is not well suited for this test image because of the additive inhomogeneity. Finally, the distortions generated through the homogenization of infinite degree could be substantially decreased by performing a prior $\mathscr{H}_{2}\{\}$ homogenization.

For the comparison of the homogenization performance, two groove textures [see Figs. 6(a) and 7(a)] and a pressed cork texture [see Fig. 8(a)] are also used. Both groove textures contain only inhomogeneities that were caused by illumination. The pressed cork texture was taken from Ref. 19 , and a Gaussian inhomogeneity $i(\mathbf{x})$ was superposed to it according to

$$
\begin{aligned}
g(\mathbf{x})=g(x, y) & =\left[-\frac{x}{(N-1) \Delta x}\right][t(\mathbf{x})+i(\mathbf{x})] \\
& + \text { const } t(\mathbf{x}) i(\mathbf{x}) \frac{x}{(N-1) \Delta x}
\end{aligned}
$$

The computation results of the inhomogeneity indicator are shown in Table 2. Because of the lower harmonic distortion, the computation of $\mathscr{H}_{2}\{\}$ was performed in the frequency domain.

Figure 6(a) shows an end-milling texture with a bright stripe. After performing a homomorphic filtering or a $\mathscr{H}_{1}\{\}$ homogenization, the stripe is still clearly visible, although the homomorphic filtering performs a little better in this case. In contrast to this, both $\mathscr{H}_{2}\{\}$ and $\mathscr{H}_{\infty}\left\{\mathscr{H}_{2}\{\} ; H_{2}(g)\right\}$ operators lead to much better results, in which hitherto hidden details become visible and the stripelike inhomogeneity can no longer be recognized.

The planed surface shown in Fig. 7(a) has a dark shadow in the upper left corner. By means of homomorphic filtering, the shadow could partially be suppressed, but it is still visible. With the $\mathscr{H}_{2}\{\}$ operator, not only could the shadow be completely suppressed, but a better homogenization of the remaining image areas could be achieved.
Finally, the pressed cork texture in Fig. 8(a) shows a transition from a pure additive inhomogeneity on the left side to a pure multiplicative one on the right side. As expected, the homomorphic filtering performs here poorly on the left side and well on the right side. The $\mathscr{H}_{2}\{\}$ operator, however, shows a good performance throughout.

In all examples, the visual impression coincides with the numerical results of the inhomogeneity indicator. We also examined many other textures and obtained similar results.

From all the cases treated, we can conclude that the $\mathscr{H}_{2}\{\}$ and $\mathscr{H}_{\infty}\left\{\mathscr{H}_{2}\{\} ; H_{2}(g)\right\}$ operators show the best overall performance. The images obtained with these two methods fulfill both requirements of very high homogeneity and low distortion. We also found that the implementation of the $\mathscr{H}_{2}\{\}$ operator in the frequency domain leads to more homogeneous and less distorted results than the computation in the space domain. In addition, for such images as those treated in this paper, the frequency domain implementation is computationally more efficient than a homogenization in space domain because of the large neighborhood set $\mathbf{U}$ necessary.

\section{Summary and Conclusion}

In this paper, we have shown that images taken from homogeneously textured surfaces are often degraded by an intensity inhomogeneity due to the acquisition process. This inhomogeneity is considered to be an irrelevant and disturbing signal component, which should be suppressed to enhance the desired texture component and to ease a subsequent image analysis.

We pointed out that, in the context of homogenization of images of textured surfaces, it is not always reasonable to assume a pure multiplicative composition of the texture and an inhomogeneity.

We introduced the notion of inhomogeneity of $n$ 'th degree based on first-order statistics. For the first and second degrees, we have deduced corresponding signal models for the composition of texture and inhomogeneity as well as suitable homogenization techniques. We have found that, regarding the visual appearance of a texture, a homogenization of second degree, which equalizes the local mean gray value and the local contrast, is mostly sufficient.

We proposed a frequency domain algorithm to implement efficiently the homogenization of second degree, keeping nonlinear distortion very low.

To achieve a still higher homogeneity level, we suggested a homogenization of second degree followed by a homogenization of infinite degree. The second homogenization step is accomplished with a sliding histogram technique. In contrast to sliding histogram methods proposed in literature, we derive the desired histogram from the given image data. Then, the gray values are manipulated with the aim of changing the local histograms into the global histogram of the second-degree homogenized image. This adaptive two-step processing keeps nonlinear distortion low and has a very high homogenization performance.

Finally, we have compared our two homogenization methods visually and quantitatively with the popular homomorphic filtering technique, which assumes a pure multiplicative inhomogeneity. We found that our methods achieve much better results for synthetic as well as for realistic images of textured surfaces. 


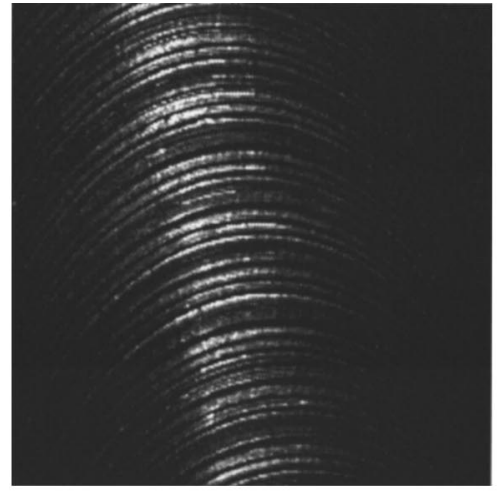

(a)

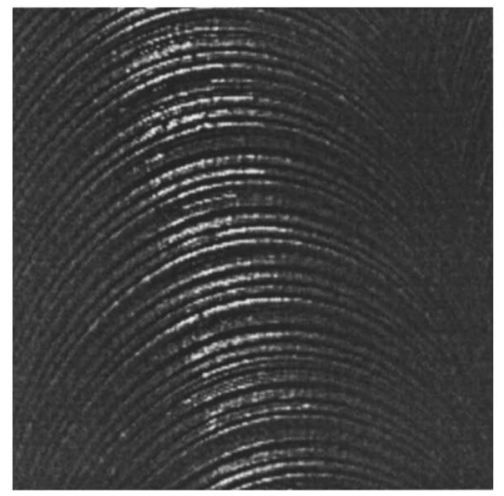

(c)

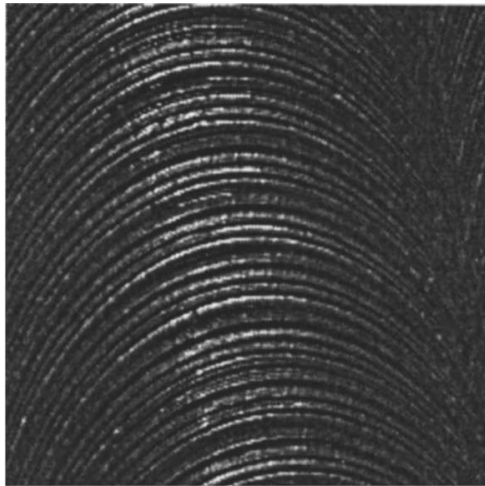

(b)

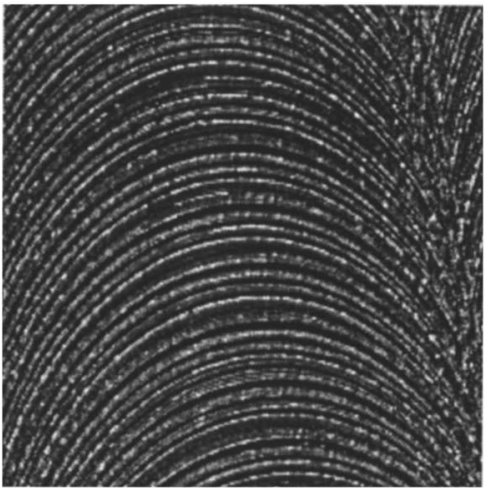

(d)

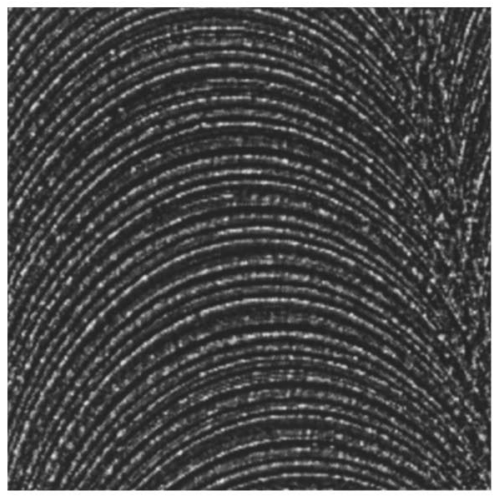

(e)

Fig. 6 (a) End-milling texture, (b) homomorphic filtering, (c) $\mathscr{H}_{1}\{\}$, (d) $\mathscr{H}_{2}\{\}$, and (e) $\mathscr{H}_{\infty}\left\{\mathscr{H}_{2}\{\}\right.$; $\left.H_{2}(g)\right\}$.

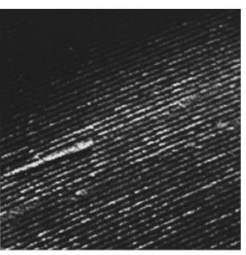

(a)

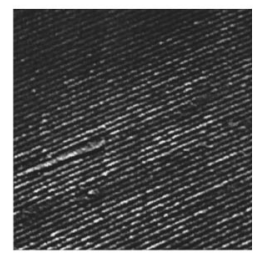

(b)

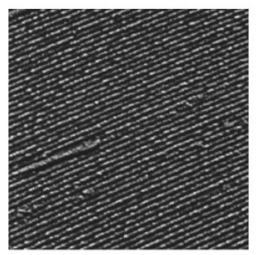

(c)

Fig. 7 (a) Planing texture, (b) homomorphic filtering, and (c) $\mathscr{H}_{2}\{\}$.

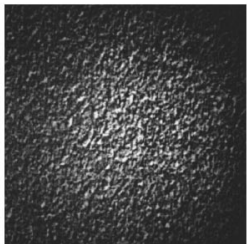

(a)

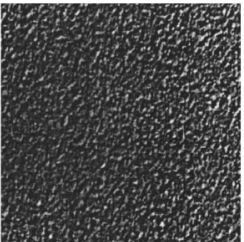

(b)

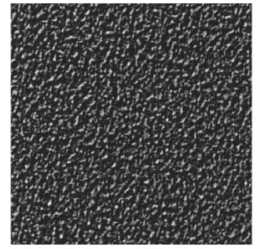

(c)
Fig. 8 (a) Pressed cork texture, (b) homomorphic filtering, and (c) $\mathscr{H}_{2}\{\}$. 
Table 2 Computation results of the inhomogeneity indicator.

\begin{tabular}{llllc}
\hline \hline & $\begin{array}{c}\text { Test } \\
\text { Image }\end{array}$ & $\begin{array}{c}\text { End-Milling } \\
\text { Texture }\end{array}$ & $\begin{array}{c}\text { Planing } \\
\text { Texture }\end{array}$ & $\begin{array}{c}\text { Pressed } \\
\text { Cork Texture }\end{array}$ \\
\hline Original & 0.46 & 1.01 & 0.69 & 0.89 \\
Homomorphic filtering & 0.18 & 0.36 & 0.33 & 0.24 \\
$\mathscr{H}_{1}\{\}$ & 0.009 & 0.40 & 0.39 & 0.19 \\
$\mathscr{H}_{2}\{\}$ & 0.009 & 0.060 & 0.046 & 0.053 \\
$\mathscr{H}_{\infty}\left\{; H_{\text {uni }}(g)\right\}$ & 0.045 & 0.048 & 0.037 & 0.030 \\
$\mathscr{H}_{\infty}\left\{\mathscr{H}_{2}\{\} ; H_{2}(g)\right\}$ & 0.009 & 0.022 & 0.018 & 0.019 \\
\hline \hline
\end{tabular}

\section{Appendix 1: Histogram Approximation with Edgeworth Series}

The Edgeworth series ${ }^{20}$ :

$$
\begin{aligned}
\hat{H}(\gamma)= & W_{\theta}(\chi)-\frac{1}{3 !}\left(\frac{\mu_{3}}{\mu_{2}^{3 / 2}}\right) W_{\theta}^{(3)}(\chi)+\frac{1}{4 !}\left(\frac{\mu_{4}}{\mu_{2}^{2}}-3\right) W_{\theta}^{(4)}(\chi) \\
& +\frac{10}{6 !}\left(\frac{\mu_{3}}{\mu_{2}^{3 / 2}}\right)^{2} W_{\theta}^{(6)}(\chi),
\end{aligned}
$$$$
\chi=\frac{\gamma-\mu_{1}}{\sqrt{\mu_{2}}}, \quad W_{\theta}(\theta)=\int_{-\infty}^{\theta} \frac{1}{\sqrt{2 \pi}} \exp \left(-\frac{\vartheta^{2}}{2}\right) \mathrm{d} \vartheta
$$

is a useful approximation to describe the desired cumulative histogram $H(g)$ needed for homogenization of infinite degree. Here $\mu_{1}$ is the expectation and $\mu_{2}$ to $\mu_{4}$ are the central moments of the distribution to be approximated. The coefficients $\mu_{3} \mu_{2}^{-1.5}$ and $\mu_{4} \mu_{2}^{-2}$ are the skewness and the kurtosis, respectively. By means of estimating the moments $\mu_{1}$ to $\mu_{4}$ globally, the Edgeworth series can be easily adapted to given image data. For unbiased estimators for $\mu_{3}$ and $\mu_{4}$, we refer to Ref. 20. An important advantage of the Edgeworth series approximation is that it depends explicitly on such well-interpretable quantities as skewness and kurtosis, which thus can be specifically manipulated. Of course, the distribution $\hat{H}(\gamma)$ must be sampled and normalized to evaluate Eq. (22).

\section{Appendix 2: Inhomogeneity Indicator}

The quantitative comparison of the presented homogenization methods is achieved by means of an inhomogeneity indicator $Q_{I}$, which must be invariant to both the global average gray level and a global contrast factor:
$Q_{I}\left\{k_{1}+k_{2} g(\mathbf{x})\right\}=Q_{I}\{g(\mathbf{x})\} \quad \forall k_{1} \in \mathbf{R}, \quad k_{2} \in \mathbf{R} \backslash\{0\}$.

The original image $g(\mathbf{x})$ is partitioned into four square windows of the same size, then each window is partitioned again into four square windows and so on (see Fig. 9). For each window $l, i$, the cumulative histogram $H_{l, i}(g)$ is computed.

Considering Eq. (29), we define an inhomogeneity indicator $Q_{I}$ as

$Q_{I}:=\frac{1}{m} \sum_{l=1}^{m} \frac{2}{\left(4^{l}-1\right) 4^{l}} \frac{1}{\hat{\sigma}_{l}} \sum_{i=1}^{4^{l}-1} \sum_{j=i+1}^{4^{l}} \mathrm{~d}\left(H_{l, i}, H_{l, j}\right)$,

$\mathrm{d}\left(H_{l, i}, H_{l, j}\right):=\sum_{\gamma=g_{1}}^{g_{G}}\left|H_{l, i}(\gamma)-H_{l, j}(\gamma)\right|$,

where $m$ must be chosen such that the smallest windows (size $N / 2^{m} \times N / 2^{m}$ ) are still much greater than the largest details of the texture $t(\mathbf{x})$.

The average empirical standard deviation $\hat{\sigma}_{l}$ in the l'th plane is

$\hat{\sigma}_{l}:=\frac{1}{4^{l}} \sum_{i=1}^{4^{l}}\left\{\sum_{\gamma=g_{1}}^{g_{G}}\left[\gamma-\sum_{\lambda=g_{1}}^{g_{G}} \lambda h_{l, i}(\lambda)\right]^{2} h_{l, i}(\gamma)\right\}^{1 / 2}$.

Indicator $Q_{I}$ is a weighted average of the $L_{1}$ distances between the local cumulative histograms at different scales.

\section{Appendix 3: Harmonic Distortion}

To compare the harmonic distortion due to the nonlinear characteristics of the homogenization operators, these are applied to a harmonic test signal (amplitude $A=50$, spatial frequency $\mathbf{f}_{0}$ ) with an additively superposed, low-frequency bandlimited inhomogeneity and an additive constant $\mu=120$. The quotient of the power of the harmonic test signal to the power of the inhomogeneity was chosen to approximately 2.4 . Figure 5 shows the resulting test image $\nu(\mathbf{x})$.

The measure of the harmonic distortion is defined as

$$
\begin{aligned}
D=D\{\mathscr{H}\{\nu(\mathbf{x})\}\}:= & \frac{\left(A_{2}^{2}+A_{3}^{2}+\cdots\right)^{1 / 2}}{A_{1}}, \\
A_{k}=A_{k}\{\mathscr{H}\{\nu(\mathbf{x})\}\}:= & \left|F\{\mathscr{H}\{\nu(\mathbf{x})\}\}\left(k \mathbf{f}_{0}\right)\right|, \\
& \mathbf{f}_{0} \approx(N \Delta x)^{-1}(15,20)^{T} .
\end{aligned}
$$

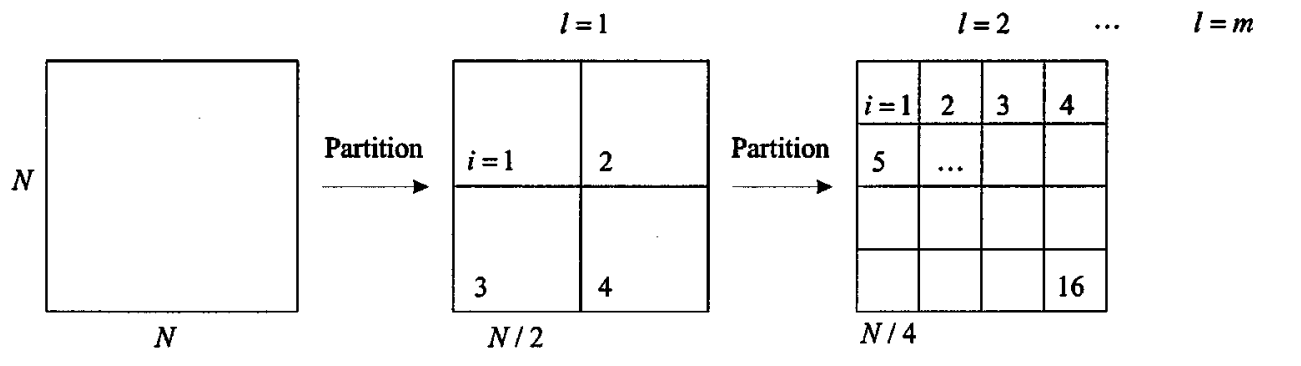

Fig. 9 Image partitioning for the evaluation of inhomogeneity. 


\section{Acknowledgments}

The authors would like to thank Doris Krahe, Dirk vom Stein, and Prof. Franz Mesch for helpful discussions and valuable comments on a draft version of this paper.

\section{References}

1. F. E. Nicodemus, J. C. Richmond, J. J. Hsia, I. W. Ginsberg, and T. Limperis, Geometrical Considerations and Nomenclature for Reflectance, NBS Monograph 160, National Bureau of Standards, U.S. Department of Commerce, Washington DC (1977).

2. B. K. P. Horn and R. W. Sjoberg, "Calculating the reflectance map," in Shape from Shading, B. K. P. Horn and M. J. Brooks, Eds., pp. 215-244, MIT Press, Cambridge, MA (1989)

3. G. Wedlich, "Ein lokaladaptives Bildverarbeitungsverfahren zum Helligkeitsausgleich von unregelmäßig ausgeleuchteten Fernsehbildern," DrIng Thesis, University of Braunschweig (1980).

4. R. C. Gonzalez and P. Wintz, Digital Image Processing, AddisonWesley, Reading, MA (1977).

5. A. K. Jain, Fundamentals of Digital Image Processing, Prentice-Hall, Englewood Cliffs, NJ (1989).

6. R. M. Haralick, K. Shanmugam, and I. Dinstein, "Textural features for image classification," IEEE Trans. Syst. Man Cybernet. SMC3(6), 610-621 (1973).

7. F. Ade, 'Characterization of textures by 'eigenfilters,' "' Signal Process. 5, 451-457 (1983)

8. N. Lins, "Beschreibung von Texturen mit Hilfe statistischer Methoden für die Anwendung bei der Segmentierung und Qualträtskontrolle," Dr Thesis No. 8270, ETH, Zürich (1987).

9. F. Tomita, S. Tsuji, Computer Analysis of Visual Textures, Kluwer Academic, Boston, Dordrecht, London (1990).

10. A. Papoulis, Probability, Random Variables and Stochastic Processes, McGraw-Hill, New York (1984).

11. F. M. Wahl, Digitale Bildsignalverarbeitung, Springer-Verlag, Berlin, Heidelberg, New York, Tokyo (1984).

12. R. H. Wallis, "An approach for the space variant restoration and enhancement of images," in Proc. Symp. on Current Mathematical Problems in Image Scenes, pp. 329-340, Monterey, CA (1976).

13. R. M. Haralick and L. G. Shapiro, Computer and Robot Vision, Vol. 1, Addison-Wesley, Reading, MA (1992).

14. J.-S. Lee, "Digital image enhancement and noise filtering by use of local statistics," IEEE Trans. Pattern Anal. Mach. Intell. PAMI-2(2), $165-168$ (1980).
15. E. O. Brigham, The Fast Fourier Transform and its Applications, Prentice-Hall, Englewood Cliffs, NJ (1988).

16. J. Beyerer, Analyse von Riefentexturen, VDI Verlag, Düsseldorf (1994).

17. J. Gauch, "Investigations of contrast space defined by variations on histogram equalization," CVGIP: Graphical Models and Image Processing 54(4), 269-280 (1992).

18. P. A. Chochia, "Image enhancement using sliding histograms," Comput. Vis. Graph. Image Process. 44, 211-229 (1988).

19. P. Brodatz, Textures, Dover Publications, New York (1966).

20. G. A. Korn and T. M. Korn, Mathematical Handbook for Scientists and Engineers, McGraw-Hill, New York (1968).

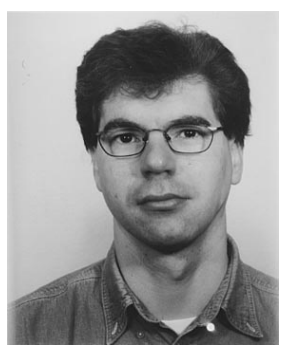

Jürgen Beyerer received an MS degree in electrical engineering in 1989 and a $\mathrm{PhD}$ degree in automated visual inspection in 1994, both from the University of Karlsruhe, Germany. He is currently a postdoctoral research associate at the Institut für Meß- und Regelungstechnik of the University of Karlsruhe. His research interests are measurement theory, information theory, statistical signal processing, image processing, and automated visual inspection.

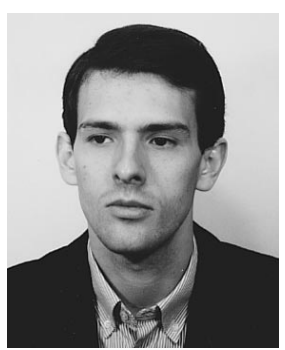

Fernando Puente León received an MS degree in electrical engineering in 1994 from the University of Karlsruhe, Germany. Since May 1994, he has been a research assistant with the Institut für Me $\beta$ und Regelungstechnik of the University of Karlsruhe. His research interests include image processing, automated visual inspection, and pattern recognition. He is currently working on the development of image processing methods for the identification of firearms. 